\title{
Notes on Intuitionistic (T, S)-Fuzzy Subfields of A Field
}

\author{
B.Anandh ${ }^{1} \&$ R.Giri ${ }^{2}$ \\ ${ }^{I}$ Department of Mathematics, H.H.The Rajah's College, Pudukkottai, Tamilnadu, India, \\ ${ }^{2}$ Research Scholar, Department of Mathematics, Sudharsan College of arts and science, Perumanadu, \\ Pudukkottai, Tamilnadu, India.
}

\begin{abstract}
In this paper, we made an attempt to study the algebraic nature of intuitionistic (T, S)-fuzzy subfield of a field.

Keywords: T-norm, S-norm, fuzzy subset, intuitionistic fuzzy subset, fuzzy subfield, intuitionistic (T, S)-fuzzy subfield, Product.
\end{abstract}

\section{Introduction}

Most of the problems in engineering, medical science, economics, environments, etc. have various uncertainties. To exceed these uncertainties, some kind of theories were given like theory of fuzzy sets, intuitionistic fuzzy sets and so on. Fuzzy set was introduction by L.A.Zadeh[23], several researchers explored on the generalization of the concept of fuzzy sets. Prof. K.T. Atanassov, a Bulgarian Engineer, introduced a new component which determines the degree of non-membership also in defining Intuitionistic fuzzy Subset( IFS ) theory. In 1983, he came across A.Kauffmann's book "Introduction to the theory of fuzzy subsets" Academic Press, New York, 1975, then he tried to introduce intuitionistic fuzzy subsets to study the properties of the new objects so defined. George Gargov named new sets as the "Intuitionistic fuzzy subsets", as their fuzzification denies the law of the excluded middle, $A \cup A^{c}=X$. This has encouraged Prof. K.T.Atanassov to continue his work on intuitionistic fuzzy subsets. The notion of fuzzy subgroups, anti-fuzzy subgroups, fuzzy fields and fuzzy linear spaces was introduced by Biswas.R [8, 9]. In this paper, we introduce the some theorems in intuitionistic (T, S)-fuzzy subfield of a field.

1. Preliminaries:

1.1 Definition: A T-norm is a binary operations $\mathrm{T}:[0,1] \times[0,1] \rightarrow[0,1]$ satisfying the following requirements;

(i) $\mathrm{T}(0, \mathrm{x})=0, \mathrm{~T}(1, \mathrm{x})=\mathrm{x}$ (boundary condition)

(ii) $\mathrm{T}(\mathrm{x}, \mathrm{y})=\mathrm{T}(\mathrm{y}, \mathrm{x})$ (commutativity)

(iii) $\mathrm{T}(\mathrm{x}, \mathrm{T}(\mathrm{y}, \mathrm{z}))=\mathrm{T}(\mathrm{T}(\mathrm{x}, \mathrm{y}), \mathrm{z})$ (associativity)

(iv) if $\mathrm{x} \leq \mathrm{y}$ and $\mathrm{w} \leq \mathrm{z}$, then $\mathrm{T}(\mathrm{x}, \mathrm{w}) \leq \mathrm{T}(\mathrm{y}, \mathrm{z})$ ( monotonicity).

1.2 Definition: A S-norm is a binary operation $S:[0,1] \times[0,1] \rightarrow[0,1]$ satisfying the following requirements;

(i) $0 \mathrm{~S} \mathrm{x}=\mathrm{x}, 1 \mathrm{~S} \mathrm{x}=1$ (boundary condition)

(ii) $\mathrm{x} S \mathrm{y}=\mathrm{y} S \mathrm{x}$ (commutativity)

(iii) $x \mathrm{~S}(\mathrm{y} S \mathrm{z})=(\mathrm{x} S \mathrm{y}) \mathrm{S} \mathrm{z}$ (associativity)

(iv) if $\mathrm{x} \leq \mathrm{y}$ and $\mathrm{w} \leq \mathrm{z}$, then $\mathrm{x} \mathrm{S} \mathrm{w} \leq \mathrm{yS} z$ ( monotonicity).

1.3 Definition: Let $X$ be a non-empty set. A fuzzy subset $A$ of $X$ is a function $A: X \rightarrow[0,1]$.

1.4 Definition: Let $(F,+, \cdot)$ be a field. A fuzzy subset A of $F$ is said to be a fuzzy subfield of $F$ if the following conditions are satisfied:

(i) $\mathrm{A}(\mathrm{x}-\mathrm{y}) \geq \min (\mathrm{A}(\mathrm{x}), \mathrm{A}(\mathrm{y}))$, for all $\mathrm{x}$ and $\mathrm{y}$ in $\mathrm{F}$,

(ii) $\mathrm{A}(\mathrm{xy}) \geq \min (\mathrm{A}(\mathrm{x}), \mathrm{A}(\mathrm{y}))$, for all $\mathrm{x}$ and $\mathrm{y}$ in $\mathrm{F}$,

(iii) $\mathrm{A}\left(\mathrm{x}^{-1}\right) \geq \mathrm{A}(\mathrm{x})$, for all $\mathrm{x}$ in $\mathrm{F}-\{0\}$, where 0 is the additive identity element of $\mathrm{F}$.

1.5 Definition: An intuitionistic fuzzy subset( IFS ) $A$ of a set $X$ is defined as an object of the form $\mathrm{A}=\left\{\left\langle\mathrm{x}, \mu_{\mathrm{A}}(\mathrm{x}), v_{\mathrm{A}}(\mathrm{x})\right\rangle / \mathrm{x} \in \mathrm{X}\right\}$, where $\mu_{\mathrm{A}}: \mathrm{X} \rightarrow[0,1]$ and $v_{\mathrm{A}}: \mathrm{X} \rightarrow[0,1]$ define the degree of membership and the degree of non-membership of the element $\mathrm{x}$ in $\mathrm{X}$ respectively and for every $\mathrm{x}$ in $\mathrm{X}$ satisfying $0 \leq \mu_{\mathrm{A}}(\mathrm{x})+v_{\mathrm{A}}(\mathrm{x}) \leq 1$. 
1.6 Example: Let $X=\{a, b, c\}$ be a set. Then $A=\{\langle a, 0.5,0.3\rangle,\langle b, 0.4,0.1\rangle,\langle c, 0.5,0.4\rangle\}$ is an intuitionistic fuzzy subset of $\mathrm{X}$.

1.7 Definition: Let $(\mathrm{F},+, \cdot)$ be a field. An intuitionistic fuzzy subset A of $\mathrm{F}$ is said to be an intuitionistic fuzzy subfield of a field $F$ with respect to (T, S)-norm ( intuitionistic (T, S)-fuzzy subfield of a field ) of $F$ if the following conditions are satisfied:

(i) $\mu_{\mathrm{A}}(\mathrm{x}+\mathrm{y}) \geq \mathrm{T}\left(\mu_{\mathrm{A}}(\mathrm{x}), \mu_{\mathrm{A}}(\mathrm{y})\right)$, for all $\mathrm{x}$ and $\mathrm{y}$ in $\mathrm{F}$,

(ii) $\mu_{\mathrm{A}}(-\mathrm{x}) \geq \mu_{\mathrm{A}}(\mathrm{x})$, for all $\mathrm{x}$ in $\mathrm{F}$,

(iii) $\mu_{\mathrm{A}}(\mathrm{xy}) \geq \mathrm{T}\left(\mu_{\mathrm{A}}(\mathrm{x}), \mu_{\mathrm{A}}(\mathrm{y})\right)$, for all $\mathrm{x}$ and $\mathrm{y}$ in $\mathrm{F}$,

(iv) $\mu_{\mathrm{A}}\left(\mathrm{x}^{-1}\right) \geq \mu_{\mathrm{A}}(\mathrm{x})$, for all $\mathrm{x} \neq 0$ in $\mathrm{F}$,

(v) $v_{A}(x+y) \leq S\left(v_{A}(x), v_{A}(y)\right)$, for all $x$ and $y$ in $F$,

(vi) $v_{A}(-x) \leq v_{A}(x)$, for all $x$ in $F$,

(vii) $v_{A}(x y) \leq S\left(v_{A}(x), v_{A}(y)\right)$, for all $x$ and $y$ in $F$,

(viii) $v_{A}\left(x^{-1}\right) \leq v_{A}(x)$, for all $x \neq 0$ in $F$.

1.8 Definition: Let $(\mathrm{F},+, \cdot)$ and $\left(\mathrm{F}^{\prime},+, \cdot\right)$ be any two fields. Let $\mathrm{f}: \mathrm{F} \rightarrow \mathrm{F}^{\prime}$ be any function and $\mathrm{A}$ be an intuitionistic fuzzy subfield in $F, V$ be an intuitionistic fuzzy subfield in $f(F)=F^{\prime}$, defined by $\mu_{\mathrm{V}}(\mathrm{y})$

$=\operatorname{Sup}_{x \in f^{-1}(y)} \mu_{\mathrm{A}}(\mathrm{x})$ and $v_{\mathrm{V}}(\mathrm{y})=\inf _{x \in f^{-1}(y)} v_{\mathrm{A}}(\mathrm{x})$, for all $\mathrm{x}$ in $\mathrm{F}$ and $\mathrm{y}$ in $\mathrm{F}^{\prime}$. Then $\mathrm{A}$ is called a preimage of $\mathrm{V}$ under $\mathrm{f}$ and is denoted by $\mathrm{f}^{-1}(\mathrm{~V})$.

1.9 Definition: Let $A$ and $B$ be any two intuitionistic fuzzy subsets of sets $\mathrm{G}$ and $\mathrm{H}$, respectively. The product of $A$ and $B$, denoted by $A \times B$, is defined as $A \times B=\left\{\left\langle(x, y), \mu_{A \times B}(x, y), v_{A \times B}(x, y)\right\rangle /\right.$ for all $x$ in $G$ and $y$ in $H\}$, where $\mu_{A \times B}(x, y)=\min \left\{\mu_{A}(x), \mu_{B}(y)\right\}$ and $v_{A \times B}(x, y)=\max \left\{v_{A}(x), v_{B}(y)\right\}$, for all $x$ in $\mathrm{G}$ and $\mathrm{y}$ in $\mathrm{H}$.

1.10 Definition: Let $A$ be an intuitionistic fuzzy subset in a set $S$, the strongest intuitionistic fuzzy relation on $S$, that is an intuitionistic fuzzy relation on $A$ is $V=\left\{\left\langle(x, y), \mu_{V}(x, y), v_{V}(x, y)\right\rangle / x\right.$ and $y$ in $S\}$ given by $\mu_{\mathrm{V}}(\mathrm{x}, \mathrm{y})=\min \left\{\mu_{\mathrm{A}}(\mathrm{x}), \mu_{\mathrm{A}}(\mathrm{y})\right\}$ and $v_{\mathrm{V}}(\mathrm{x}, \mathrm{y})=\max \left\{v_{\mathrm{A}}(\mathrm{x}), v_{\mathrm{A}}(\mathrm{y})\right\}$, for all $\mathrm{x}$ and $\mathrm{y}$ in $\mathrm{S}$.

1.11 Definition: Let $A$ be an intuitionistic fuzzy subfield of a field $(F,+, \cdot)$ and a in $F$. Then the pseudo intuitionistic fuzzy coset $(a A)^{p}$ is defined by $\left(\left(a \mu_{A}\right)^{p}\right)(x)=p(a) \mu_{A}(x)$ and $\left(\left(a v_{A}\right)^{p}\right)(x)=$ $p(a) v_{A}(x)$, for every $x$ in $F$ and for some $p$ in $P$.

\section{Properties of Intuitionistic (T, S)-Fuzzy Subfields}

2.1 Theorem: If $A$ and $B$ are intuitionistic (T, S)-fuzzy subfields of the fields $\mathrm{G}$ and $\mathrm{H}$ respectively, then $A \times B$ is an intuitionistic $(T, S)$-fuzzy subfield of $G \times H$.

Proof: Let $A$ and $B$ be intuitionistic $(T, S)$-fuzzy subfields of the fields $G$ and $H$ respectively. Let $x_{1}$ and $x_{2}$ be in $G, y_{1}$ and $y_{2}$ be in $H$. Then $\left(x_{1}, y_{1}\right)$ and $\left(x_{2}, y_{2}\right)$ are in $G \times H$. Now, $\mu_{A \times B}\left[\left(x_{1}, y_{1}\right)-\left(x_{2}, y_{2}\right)\right]=$ $\mu_{\mathrm{A} \times \mathrm{B}}\left(\mathrm{x}_{1}-\mathrm{x}_{2}, \mathrm{y}_{1}-\mathrm{y}_{2}\right)=\min \left\{\mu_{\mathrm{A}}\left(\mathrm{x}_{1}-\mathrm{x}_{2}\right), \mu_{\mathrm{B}}\left(\mathrm{y}_{1}-\mathrm{y}_{2}\right)\right\} \geq \min \left\{\mathrm{T}\left(\mu_{\mathrm{A}}\left(\mathrm{x}_{1}\right), \mu_{\mathrm{A}}\left(\mathrm{x}_{2}\right)\right), \mathrm{T}\left(\mu_{\mathrm{B}}\left(\mathrm{y}_{1}\right), \mu_{\mathrm{B}}\left(\mathrm{y}_{2}\right)\right)\right\} \geq$ $\mathrm{T}\left(\min \left(\mu_{\mathrm{A}}\left(\mathrm{x}_{1}\right), \mu_{\mathrm{B}}\left(\mathrm{y}_{1}\right)\right), \min \left(\mu_{\mathrm{A}}\left(\mathrm{x}_{2}\right), \mu_{\mathrm{B}}\left(\mathrm{y}_{2}\right)\right)\right)=\mathrm{T}\left(\mu_{\mathrm{A} \times \mathrm{B}}\left(\mathrm{x}_{1}, \mathrm{y}_{1}\right), \mu_{\mathrm{A} \times \mathrm{B}}\left(\mathrm{x}_{2}, \mathrm{y}_{2}\right)\right)$.

Therefore, $\mu_{\mathrm{A} \times \mathrm{B}}\left[\left(\mathrm{x}_{1}, \mathrm{y}_{1}\right)-\left(\mathrm{x}_{2}, \mathrm{y}_{2}\right)\right] \geq \mathrm{T}\left(\mu_{\mathrm{A} \times \mathrm{B}}\left(\mathrm{x}_{1}, \mathrm{y}_{1}\right), \mu_{\mathrm{A} \times \mathrm{B}}\left(\mathrm{x}_{2}, \mathrm{y}_{2}\right)\right)$, for all $\mathrm{x}_{1}$ and $\mathrm{x}_{2}$ in $\mathrm{G}$ and $\mathrm{y}_{1}$ and $\mathrm{y}_{2}$ in H. Now, $\mu_{\mathrm{A} \times \mathrm{B}}\left[\left(\mathrm{x}_{1}, \mathrm{y}_{1}\right)\left(\mathrm{x}_{2}, \mathrm{y}_{2}\right)^{-1}\right]=\mu_{\mathrm{A} \times \mathrm{B}}\left(\mathrm{x}_{1} \mathrm{x}_{2}{ }^{-1}, \mathrm{y}_{1} \mathrm{y}_{2}^{-1}\right)=\min \left\{\mu_{\mathrm{A}}\left(\mathrm{x}_{1} \mathrm{x}_{2}{ }^{-1}\right), \mu_{\mathrm{B}}\left(\mathrm{y}_{1} \mathrm{y}_{2}{ }^{-1}\right)\right\} \geq \min \{\mathrm{T}($ $\left.\left.\mu_{\mathrm{A}}\left(\mathrm{x}_{1}\right), \mu_{\mathrm{A}}\left(\mathrm{x}_{2}\right)\right), \mathrm{T}\left(\mu_{\mathrm{B}}\left(\mathrm{y}_{1}\right), \mu_{\mathrm{B}}\left(\mathrm{y}_{2}\right)\right)\right\} \geq \mathrm{T}\left(\min \left(\mu_{\mathrm{A}}\left(\mathrm{x}_{1}\right), \mu_{\mathrm{B}}\left(\mathrm{y}_{1}\right)\right), \min \left(\mu_{\mathrm{A}}\left(\mathrm{x}_{2}\right), \mu_{\mathrm{B}}\left(\mathrm{y}_{2}\right)\right)\right)=\mathrm{T}\left(\mu_{\mathrm{A} \times \mathrm{B}}\left(\mathrm{x}_{1}, \mathrm{y}_{1}\right)\right.$, $\left.\mu_{\mathrm{A} \times \mathrm{B}}\left(\mathrm{x}_{2}, \mathrm{y}_{2}\right)\right)$. Therefore, $\mu_{\mathrm{A} \times \mathrm{B}}\left[\left(\mathrm{x}_{1}, \mathrm{y}_{1}\right)\left(\mathrm{x}_{2}, \mathrm{y}_{2}\right)^{-1}\right] \geq \mathrm{T}\left(\mu_{\mathrm{A} \times \mathrm{B}}\left(\mathrm{x}_{1}, \mathrm{y}_{1}\right), \mu_{\mathrm{A} \times \mathrm{B}}\left(\mathrm{x}_{2}, \mathrm{y}_{2}\right)\right)$, for all $\mathrm{x}_{1}$ and $\mathrm{x}_{2} \neq 0$ in $\mathrm{G}$ and $\mathrm{y}_{1}$ and $\mathrm{y}_{2} \neq 0^{\prime}$ in $\mathrm{H}$. And, $\mathrm{v}_{\mathrm{A} \times \mathrm{B}}\left[\left(\mathrm{x}_{1}, \mathrm{y}_{1}\right)-\left(\mathrm{x}_{2}, \mathrm{y}_{2}\right)\right]=v_{\mathrm{A} \times \mathrm{B}}\left(\mathrm{x}_{1}-\mathrm{x}_{2}, \mathrm{y}_{1}-\mathrm{y}_{2}\right)=\max \left\{\mathrm{v}_{\mathrm{A}}\left(\mathrm{x}_{1}-\mathrm{x}_{2}\right), v_{\mathrm{B}}\left(\mathrm{y}_{1}-\mathrm{y}_{2}\right)\right\}$ $\leq \max \left\{\mathrm{S}\left(\mathrm{v}_{\mathrm{A}}\left(\mathrm{x}_{1}\right), v_{\mathrm{A}}\left(\mathrm{x}_{2}\right)\right), \mathrm{S}\left(\mathrm{v}_{\mathrm{B}}\left(\mathrm{y}_{1}\right), v_{\mathrm{B}}\left(\mathrm{y}_{2}\right)\right)\right\} \leq \mathrm{S}\left(\max \left(\mathrm{v}_{\mathrm{A}}\left(\mathrm{x}_{1}\right), v_{\mathrm{B}}\left(\mathrm{y}_{1}\right)\right), \max \left(\mathrm{v}_{\mathrm{A}}\left(\mathrm{x}_{2}\right), v_{\mathrm{B}}\left(\mathrm{y}_{2}\right)\right)\right)=$ $\mathrm{S}\left(v_{\mathrm{A} \times \mathrm{B}}\left(\mathrm{x}_{1}, \mathrm{y}_{1}\right), v_{\mathrm{A} \times \mathrm{B}}\left(\mathrm{x}_{2}, \mathrm{y}_{2}\right)\right)$. Therefore, $v_{\mathrm{A} \times \mathrm{B}}\left[\left(\mathrm{x}_{1}, \mathrm{y}_{1}\right)-\left(\mathrm{x}_{2}, \mathrm{y}_{2}\right)\right] \leq \mathrm{S}\left(\mathrm{v}_{\mathrm{A} \times \mathrm{B}}\left(\mathrm{x}_{1}, \mathrm{y}_{1}\right), v_{\mathrm{A} \times \mathrm{B}}\left(\mathrm{x}_{2}, \mathrm{y}_{2}\right)\right)$, for all $\mathrm{x}_{1}$, $\mathrm{x}_{2}$ in $\mathrm{G}$ and $\mathrm{y}_{1}$ and $\mathrm{y}_{2}$ in $\mathrm{H}$. And, $v_{\mathrm{A} \times \mathrm{B}}\left[\left(\mathrm{x}_{1}, \mathrm{y}_{1}\right)\left(\mathrm{x}_{2}, \mathrm{y}_{2}\right)^{-1}\right]=v_{\mathrm{A} \times \mathrm{B}}\left(\mathrm{x}_{1} \mathrm{x}_{2}^{-1}, \mathrm{y}_{1} \mathrm{y}_{2}^{-1}\right)=\max \left\{v_{\mathrm{A}}\left(\mathrm{x}_{1} \mathrm{x}_{2}{ }^{-1}\right), v_{\mathrm{B}}\left(\mathrm{y}_{1} \mathrm{y}_{2}^{-}\right.\right.$ $\left.\left.{ }^{1}\right)\right\} \leq \max \left(S\left(v_{A}\left(x_{1}\right), v_{A}\left(x_{2}\right)\right), S\left(v_{B}\left(y_{1}\right), v_{B}\left(y_{2}\right)\right)\right) \leq S\left(\max \left(v_{A}\left(x_{1}\right), v_{B}\left(y_{1}\right)\right), \max \left(v_{A}\left(x_{2}\right), v_{B}\left(y_{2}\right)\right)\right)=$ $\mathrm{S}\left(\mathrm{v}_{\mathrm{A} \times \mathrm{B}}\left(\mathrm{x}_{1}, \mathrm{y}_{1}\right), v_{\mathrm{A} \times \mathrm{B}}\left(\mathrm{x}_{2}, \mathrm{y}_{2}\right)\right)$. Therefore, $v_{\mathrm{A} \times \mathrm{B}}\left[\left(\mathrm{x}_{1}, \mathrm{y}_{1}\right)\left(\mathrm{x}_{2}, \mathrm{y}_{2}\right)^{-1}\right] \leq \mathrm{S}\left(\mathrm{v}_{\mathrm{A} \times \mathrm{B}}\left(\mathrm{x}_{1}, \mathrm{y}_{1}\right), v_{\mathrm{A} \times \mathrm{B}}\left(\mathrm{x}_{2}, \mathrm{y}_{2}\right)\right)$, for all $\mathrm{x}_{1}$ and $x_{2} \neq 0$ in $G$ and $y_{1}$ and $y_{2} \neq 0$ in $H$. Hence $A \times B$ is an intuitionistic $(T, S)$-fuzzy subfield of $G \times H$.

2.2 Theorem: Let A and B be intuitionistic fuzzy subsets of the fields $G$ and $H$ respectively. Suppose that 0,1 and $0^{\prime}, 1^{\prime}$ are the identity elements of $\mathrm{G}$ and $\mathrm{H}$ respectively. If $\mathrm{A} \times \mathrm{B}$ is an intuitionistic (T, S)fuzzy subfield of $\mathrm{G} \times \mathrm{H}$, then at least one of the following two statements must hold. 
(i) $\mu_{B}\left(0^{\prime}\right) \geq \mu_{A}(x)$, for all $x$ in $G$ and $\mu_{B}\left(1^{\prime}\right) \geq \mu_{A}(x)$, for all $x \neq 0$ in $G$ and $v_{B}\left(0^{\prime}\right) \leq v_{A}(x)$, for all $x$ in $G$ and $v_{\mathrm{B}}\left(1^{\prime}\right) \leq v_{\mathrm{A}}(\mathrm{x})$, for all $\mathrm{x} \neq 0$ in $\mathrm{G}$,

(ii) $\mu_{\mathrm{A}}(0) \geq \mu_{\mathrm{B}}(\mathrm{y})$, for all $\mathrm{y}$ in $\mathrm{H}$ and $\mu_{\mathrm{A}}(1) \geq \mu_{\mathrm{B}}(\mathrm{y})$, for all $\mathrm{y} \neq 0^{\prime}$ in $\mathrm{H}$ and $v_{\mathrm{A}}(0) \leq v_{\mathrm{B}}(\mathrm{y})$, for all $\mathrm{y}$ in $\mathrm{H}$ and $v_{\mathrm{A}}(1) \leq v_{\mathrm{B}}(\mathrm{y})$, for all $\mathrm{y} \neq 0^{\prime}$ in $\mathrm{H}$.

Proof: Let $\mathrm{A} \times \mathrm{B}$ is an intuitionistic $(\mathrm{T}, \mathrm{S})$-fuzzy subfield of $\mathrm{G} \times \mathrm{H}$.

By contraposition, suppose that none of the statements (i) and (ii) holds.

Then we can find $\mathrm{a}$ in $\mathrm{G}$ and $\mathrm{b}$ in $\mathrm{H}$ such that $\mu_{\mathrm{A}}(\mathrm{a})>\mu_{\mathrm{B}}\left(0^{\prime}\right), v_{\mathrm{A}}(\mathrm{a})<v_{\mathrm{B}}\left(0^{\prime}\right)$ and $\mu_{\mathrm{B}}(\mathrm{b})>\mu_{\mathrm{A}}(0), v_{\mathrm{B}}(\mathrm{b})<$ $v_{A}(0)$ and we can find $a \neq 0$ in $G$ and $b \neq 0$ in $H$ such that $\mu_{A}(a)>\mu_{B}\left(1^{\prime}\right), v_{A}(a)<v_{B}\left(1^{\prime}\right)$ and $\mu_{B}(b)>$ $\mu_{A}(1), v_{B}(b)<v_{A}(1)$. We have, $\mu_{A \times B}(a, b)=\min \left\{\mu_{A}(a), \mu_{B}(b)\right\}>\min \left\{\mu_{A}(0), \mu_{B}\left(0^{\prime}\right)\right\}=\mu_{A \times B}\left(0,0^{\prime}\right)$. And, $\mu_{A \times B}(a, b)=\min \left\{\mu_{A}(a), \mu_{B}(b)\right\}>\min \left\{\mu_{A}(1), \mu_{B}\left(1^{\prime}\right)\right\}=\mu_{A \times B}\left(1,1^{\prime}\right)$. We have, $v_{A \times B}(a, b)=$ $\max \left\{v_{A}(a), v_{B}(b)\right\}<\max \left\{v_{A}(0), v_{B}\left(0^{\prime}\right)\right\}=v_{A \times B}\left(0,0^{\prime}\right)$. And, $v_{A \times B}(a, b)=\max \left\{v_{A}(a), v_{B}(b)\right\}<$ $\max \left\{v_{A}(1), v_{B}\left(1^{\prime}\right)\right\}=v_{A \times B}\left(1,1^{\prime}\right)$. Thus $A \times B$ is not an intuitionistic (T, S)-fuzzy subfield of $G \times H$. Hence either $\mu_{B}\left(0^{\prime}\right) \geq \mu_{A}(x)$, for all $x$ in $G$ and $\mu_{B}\left(1^{\prime}\right) \geq \mu_{A}(x)$, for all $x \neq 0$ in $G$ and $v_{B}\left(0^{\prime}\right) \leq v_{A}(x)$, for all $x$ in $G$ and $v_{B}\left(1^{\prime}\right) \leq v_{A}(x)$, for all $x \neq 0$ in $G$ or $\mu_{A}(0) \geq \mu_{B}(y)$, for all $y$ in $H$ and $\mu_{A}(1) \geq \mu_{B}(y)$, for all $y \neq 0$ in $H$ and $v_{A}(0) \leq v_{B}(y)$, for all $y$ in $H$ and $v_{A}(1) \leq v_{B}(y)$, for all $y \neq 0^{1}$ in $H$.

2.3 Theorem: Let $A$ and $B$ be intuitionistic fuzzy subsets of the fields $G$ and $H$, respectively and $A \times B$ is an intuitionistic (T, S)-fuzzy subfield of $\mathrm{G} \times \mathrm{H}$. Then the following are true:

(i) if $\mu_{A}(x) \leq \mu_{B}\left(0^{\prime}\right)$, for all $x$ in $G$ and $\mu_{A}(x) \leq \mu_{B}\left(1^{\prime}\right)$, for all $x \neq 0$ in $G$ and $v_{A}(x) \geq v_{B}\left(0^{\prime}\right)$, for all $x$ in $G$ and $v_{A}(x) \geq v_{B}\left(1^{\prime}\right)$, for all $x \neq 0$ in $G$, then $A$ is an intuitionistic (T, S)-fuzzy subfield of $G$, where $0^{\prime}, 1^{\prime}$ are identity elements of $\mathrm{H}$.

(ii) if $\mu_{B}(x) \leq \mu_{A}(0)$ for all $x$ in $H$ and $\mu_{B}(x) \leq \mu_{A}(1)$, for all $x \neq 0$ in $H$ and $v_{B}(x) \geq v_{A}(0)$, for all $x$ in $H$ and $v_{B}(x) \geq v_{A}(1)$, for all $x \neq 0$ in $H$, then $B$ is an intuitionistic (T, S)-fuzzy subfield of $H$, where 0,1 are identity elements of $\mathrm{G}$.

(iii) either $A$ is an intuitionistic (T, S)-fuzzy subfield of $G$ or B is an intuitionistic (T, S)-fuzzy subfield of $\mathrm{H}$, where 0,1 and $0^{\prime}, 1^{\prime}$ are the identity elements of $\mathrm{G}$ and $\mathrm{H}$ respectively.

Proof: Let $\mathrm{A} \times \mathrm{B}$ be an intuitionistic (T, S)-fuzzy subfield of $\mathrm{G} \times \mathrm{H}$ and $\mathrm{x}$ and $\mathrm{y}$ in $\mathrm{G}$. Then (x, $\left.0^{\prime}\right),\left(\mathrm{x}, 1^{\prime}\right)$ and $\left(\mathrm{y}, 0^{\prime}\right),\left(\mathrm{y}, 1^{\prime}\right)$ are in $\mathrm{G} \times \mathrm{H}$. Now, using the property if $\mu_{\mathrm{A}}(\mathrm{x}) \leq \mu_{\mathrm{B}}\left(0^{\prime}\right)$, for all $\mathrm{x}$ in $\mathrm{G}$ and $\mu_{\mathrm{A}}(\mathrm{x}) \leq$ $\mu_{B}\left(1^{\prime}\right)$, for all $x \neq 0$ in $G$ and $v_{A}(x) \geq v_{B}\left(0^{\prime}\right)$, for all $x$ in $G$ and $v_{A}(x) \geq v_{B}\left(1^{\prime}\right)$, for all $x \neq 0$ in $G$, where 0 and 1 are identity elements of $G$ and 0 'and 1 'are identity elements of $H$, we get $\mu_{A}(x-y)=$ $\min \left\{\mu_{\mathrm{A}}(\mathrm{x}-\mathrm{y}), \mu_{\mathrm{B}}\left(0^{\prime}+0^{\prime}\right)\right\}=\mu_{\mathrm{A} \times \mathrm{B}}\left((\mathrm{x}-\mathrm{y}),\left(0^{\prime}+0^{\prime}\right)\right)=\mu_{\mathrm{A} \times \mathrm{B}}\left[\left(\mathrm{x}, 0^{\prime}\right)+\left(-\mathrm{y}, 0^{\prime}\right)\right] \geq \min \left\{\mu_{\mathrm{A} \times \mathrm{B}}\left(\mathrm{x}, 0^{\prime}\right), \mu_{\mathrm{A} \times \mathrm{B}}(-\mathrm{y}\right.$, $\left.\left.0^{\prime}\right)\right\}=\min \left\{\mathrm{T}\left(\mu_{\mathrm{A}}(\mathrm{x}), \mu_{\mathrm{B}}\left(0^{\prime}\right)\right), \mathrm{T}\left(\mu_{\mathrm{A}}(-\mathrm{y}), \mu_{\mathrm{B}}\left(0^{\prime}\right)\right)\right\}=\mathrm{T}\left(\mu_{\mathrm{A}}(\mathrm{x}), \mu_{\mathrm{A}}(-\mathrm{y})\right) \geq \mathrm{T}\left(\mu_{\mathrm{A}}(\mathrm{x}), \mu_{\mathrm{A}}(\mathrm{y})\right)$. Therefore, $\mu_{\mathrm{A}}(\mathrm{x}-\mathrm{y}) \geq \mathrm{T}\left(\mu_{\mathrm{A}}(\mathrm{x}), \mu_{\mathrm{A}}(\mathrm{y})\right)$, for all $\mathrm{x}$ and $\mathrm{y}$ in $\mathrm{G}$. And $\mu_{\mathrm{A}}\left(\mathrm{xy}^{-1}\right)=\min \left\{\mu_{\mathrm{A}}\left(\mathrm{xy}^{-1}\right), \mu_{\mathrm{B}}\left(1^{1} 1^{1}\right)\right\}=\mu_{\mathrm{A} \times \mathrm{B}}\left(\left(\mathrm{xy}^{-1}\right)\right.$, $\left.\left(1^{\prime} 1^{\prime}\right)\right)=\mu_{\mathrm{A} \times \mathrm{B}}\left[\left(\mathrm{x}, 1^{\prime}\right)\left(\mathrm{y}^{-1}, 1^{\prime}\right)\right] \geq \mathrm{T}\left(\mu_{\mathrm{A} \times \mathrm{B}}\left(\mathrm{x}, 1^{\prime}\right), \mu_{\mathrm{A} \times \mathrm{B}}\left(\mathrm{y}^{-1}, 1^{\prime}\right)\right)=\mathrm{T}\left(\min \left(\mu_{\mathrm{A}}(\mathrm{x}), \mu_{\mathrm{B}}\left(1^{\prime}\right)\right), \min \left(\mu_{\mathrm{A}}\left(\mathrm{y}^{-1}\right)\right.\right.$, $\left.\mu_{B}\left(1^{\prime}\right)\right)=T\left(\mu_{A}(x), \mu_{A}\left(y^{-1}\right) \geq T\left(\mu_{A}(x), \mu_{A}(y)\right)\right.$. Therefore, $\mu_{A}\left(x^{-1}\right) \geq T\left(\mu_{A}(x), \mu_{A}(y)\right)$, for all $x$ and $y \neq 0$ in G. And, $\quad v_{\mathrm{A}}(\mathrm{x}-\mathrm{y})=\max \left\{v_{\mathrm{A}}(\mathrm{x}-\mathrm{y}), \quad v_{\mathrm{B}}\left(0^{\prime}+0^{\prime}\right)\right\}=v_{\mathrm{A} \times \mathrm{B}}\left((\mathrm{x}-\mathrm{y}), \quad\left(0^{\prime}+0^{\prime}\right)\right)=v_{\mathrm{A} \times \mathrm{B}}\left[\left(\mathrm{x}, 0^{\prime}\right)+\left(-\mathrm{y}, \quad 0^{\prime}\right)\right]$ $\leq \mathrm{S}\left(v_{\mathrm{A} \times \mathrm{B}}\left(\mathrm{x}, 0^{\prime}\right), v_{\mathrm{A} \times \mathrm{B}}\left(-\mathrm{y}, 0^{\prime}\right)\right)=\mathrm{S}\left(\max \left\{v_{\mathrm{A}}(\mathrm{x}), v_{\mathrm{B}}\left(0^{\prime}\right)\right\}, \max \left\{v_{\mathrm{A}}(-\mathrm{y}), v_{\mathrm{B}}\left(0^{\prime}\right)\right\}\right)=\mathrm{S}\left(v_{\mathrm{A}}(\mathrm{x}), v_{\mathrm{A}}(-\mathrm{y})\right) \leq$ $\mathrm{S}\left(v_{\mathrm{A}}(\mathrm{x}), v_{\mathrm{A}}(\mathrm{y})\right)$. Therefore, $v_{\mathrm{A}}(\mathrm{x}-\mathrm{y}) \leq \mathrm{S}\left(v_{\mathrm{A}}(\mathrm{x}), v_{\mathrm{A}}(\mathrm{y})\right)$, for all $\mathrm{x}$ and $\mathrm{y}$ in $\mathrm{G}$. And, $v_{\mathrm{A}}\left(\mathrm{xy}^{-1}\right)=$ $\max \left\{v_{\mathrm{A}}\left(\mathrm{xy}^{-1}\right), v_{\mathrm{B}}\left(1^{\prime} 1^{\prime}\right)\right\}=v_{\mathrm{A} \times \mathrm{B}}\left(\left(x y^{-1}\right),\left(1^{\prime} 1^{\prime}\right)\right)=v_{\mathrm{A} \times \mathrm{B}}\left[\left(\mathrm{x}, 1^{\prime}\right)\left(\mathrm{y}^{-1}, 1^{\prime}\right)\right] \leq \mathrm{S}\left(v_{\mathrm{A} \times \mathrm{B}}\left(\mathrm{x}, 1^{\prime}\right), v_{\mathrm{A} \times \mathrm{B}}\left(\mathrm{y}^{-1}, 1^{\prime}\right)\right)=$ $\mathrm{S}\left(\max \left(v_{\mathrm{A}}(\mathrm{x}), v_{\mathrm{B}}\left(1^{\prime}\right)\right), \max \left(v_{\mathrm{A}}\left(\mathrm{y}^{-1}\right), v_{\mathrm{B}}\left(1^{1}\right)\right)\right)=\mathrm{S}\left(v_{\mathrm{A}}(\mathrm{x}), v_{\mathrm{A}}\left(\mathrm{y}^{-1}\right)\right) \leq \mathrm{S}\left(v_{\mathrm{A}}(\mathrm{x}), v_{\mathrm{A}}(\mathrm{y})\right)$. Therefore, $v_{\mathrm{A}}\left(\mathrm{xy}^{-1}\right)$ $\leq \mathrm{S}\left(v_{\mathrm{A}}(\mathrm{x}), v_{\mathrm{A}}(\mathrm{y})\right)$, for all $\mathrm{x}$ and $\mathrm{y} \neq 0$ in $\mathrm{G}$. Hence $\mathrm{A}$ is an intuitionistic $(\mathrm{T}, \mathrm{S})$-fuzzy subfield of $\mathrm{G}$. Thus (i) is proved. Now, using the property $\mu_{B}(x) \leq \mu_{A}(0)$ for all $x$ in $H$ and $\mu_{B}(x) \leq \mu_{A}(1)$, for all $x \neq 0$ ' in $H$ and $v_{B}(x) \geq v_{A}(0)$, for all $x$ in $H$ and $v_{B}(x) \geq v_{A}(1)$, for all $x \neq 0^{\prime}$ in $H$, we get, $\mu_{B}(x-y)=\min \left\{\mu_{B}(x-y)\right.$, $\left.\mu_{\mathrm{A}}(0+0)\right\}=\mu_{\mathrm{A} \times \mathrm{B}}((0+0),(\mathrm{x}-\mathrm{y}))=\mu_{\mathrm{A} \times \mathrm{B}}[(0, \mathrm{x})+(0,-\mathrm{y})] \geq \mathrm{T}\left(\mu_{\mathrm{A} \times \mathrm{B}}(0, \mathrm{x}), \mu_{\mathrm{A} \times \mathrm{B}}(0,-\mathrm{y})\right)=\mathrm{T}\left(\min \left(\mu_{\mathrm{A}}(0), \mu_{\mathrm{B}}(\mathrm{x})\right.\right.$ ), $\left.\min \left(\mu_{\mathrm{A}}(0), \mu_{\mathrm{B}}(-\mathrm{y})\right)\right)=\mathrm{T}\left(\mu_{\mathrm{B}}(\mathrm{x}), \mu_{\mathrm{B}}(-\mathrm{y})\right) \geq \mathrm{T}\left(\mu_{\mathrm{B}}(\mathrm{x}), \mu_{\mathrm{B}}(\mathrm{y})\right)$. Therefore, $\mu_{\mathrm{B}}(\mathrm{x}-\mathrm{y}) \geq \mathrm{T}\left(\mu_{\mathrm{B}}(\mathrm{x}), \mu_{\mathrm{B}}(\mathrm{y})\right)$, for all $x, y$ in $H$. And $\mu_{B}\left(x^{-1}\right)=\min \left\{\mu_{B}\left(x^{-1}\right), \mu_{A}(1.1)\right\}=\mu_{A \times B}\left((1.1),\left(x^{-1}\right)\right)=\mu_{A \times B}\left[(1, x)\left(1, y^{-1}\right)\right] \geq$ $\mathrm{T}\left(\mu_{\mathrm{A} \times \mathrm{B}}(1, \mathrm{x}), \mu_{\mathrm{A} \times \mathrm{B}}\left(1, \mathrm{y}^{-1}\right)\right)=\mathrm{T}\left(\min \left(\mu_{\mathrm{A}}(1), \mu_{\mathrm{B}}(\mathrm{x})\right), \min \left(\mu_{\mathrm{A}}(1), \mu_{\mathrm{B}}\left(\mathrm{y}^{-1}\right)\right)\right)=\mathrm{T}\left(\mu_{\mathrm{B}}(\mathrm{x}), \mu_{\mathrm{B}}\left(\mathrm{y}^{-1}\right)\right) \geq \mathrm{T}\left(\mu_{\mathrm{B}}(\mathrm{x})\right.$, $\left.\mu_{\mathrm{B}}(\mathrm{y})\right)$. Therefore, $\mu_{\mathrm{B}}\left(\mathrm{xy}^{-1}\right) \geq \mathrm{T}\left(\mu_{\mathrm{B}}(\mathrm{x}), \mu_{\mathrm{B}}(\mathrm{y})\right)$, for all $\mathrm{x}$ and $\mathrm{y} \neq 0^{\prime}$ in $\mathrm{H}$. And, $v_{\mathrm{B}}(\mathrm{x}-\mathrm{y})=\max \left\{v_{\mathrm{B}}(\mathrm{x}-\mathrm{y})\right.$, $\left.v_{A}(0+0)\right\}=v_{A \times B}((0+0),(x-y))=v_{A \times B}[(0, x)+(0,-y)] \leq S\left(v_{A \times B}(0, x), v_{A \times B}(0,-y)\right)=S\left(\max \left(v_{A}(0)\right.\right.$, $\left.\left.v_{B}(x)\right), \max \left(v_{A}(0), v_{B}(-y)\right)\right)=S\left(v_{B}(x), v_{B}(-y)\right) \leq S\left(v_{B}(x), v_{B}(y)\right)$. Therefore, $v_{B}(x-y) \leq S\left(v_{B}(x)\right.$, $\left.v_{B}(y)\right)$, for all $x, y$ in $H$. And, $v_{B}\left(x y^{-1}\right)=\max \left(v_{B}\left(x y^{-1}\right), v_{A}(1.1)\right)=v_{A \times B}\left((1.1),\left(x y^{-1}\right)\right)$ $=v_{A \times B}\left[(1, x)\left(1, y^{-1}\right)\right] \leq S\left(v_{A \times B}(1, x), v_{A \times B}\left(1, y^{-1}\right)\right)=S\left(\max \left(v_{A}(1), v_{B}(x)\right), \max \left(v_{A}(1), v_{B}\left(y^{-1}\right)\right)\right)=$ $\mathrm{S}\left(v_{\mathrm{B}}(\mathrm{x}), v_{\mathrm{B}}\left(\mathrm{y}^{-1}\right)\right) \leq \mathrm{S}\left(v_{\mathrm{B}}(\mathrm{x}), v_{\mathrm{B}}(\mathrm{y})\right)$. Therefore, $v_{\mathrm{B}}\left(\mathrm{xy}^{-1}\right) \leq \mathrm{S}\left(v_{\mathrm{B}}(\mathrm{x}), v_{\mathrm{B}}(\mathrm{y})\right)$, for all $\mathrm{x}$ and $\mathrm{y} \neq 0^{\prime}$ in $\mathrm{H}$. Hence $B$ is an intuitionistic (T, S)-fuzzy subfield of $H$. Thus (ii) is proved. Hence (iii) is clear. 
2.4 Theorem: Let $\mathrm{A}$ be an intuitionistic fuzzy subset of a field $(\mathrm{F},+,$.$) and \mathrm{V}$ be the strongest intuitionistic fuzzy relation of $F$. Then $A$ is an intuitionistic ( $T, S$ )-fuzzy subfield of $F$ if and only if $V$ is an intuitionistic (T, S)-fuzzy subfield of F×F.

Proof: Suppose that A is an intuitionistic (T, S)-fuzzy subfield of $F$. Then for any $x=\left(x_{1}, x_{2}\right)$ and $y=$ $\left(\mathrm{y}_{1}, \mathrm{y}_{2}\right)$ are in F $\times \mathrm{F}$. We have, $\mu_{\mathrm{V}}(\mathrm{x}-\mathrm{y})=\mu_{\mathrm{V}}\left[\left(\mathrm{x}_{1}, \mathrm{x}_{2}\right)-\left(\mathrm{y}_{1}, \mathrm{y}_{2}\right)\right]=\mu_{\mathrm{V}}\left(\mathrm{x}_{1}-\mathrm{y}_{1}, \mathrm{x}_{2}-\mathrm{y}_{2}\right)=\min \left\{\mu_{\mathrm{A}}\left(\mathrm{x}_{1}-\mathrm{y}_{1}\right)\right.$, $\left.\mu_{\mathrm{A}}\left(\mathrm{x}_{2}-\mathrm{y}_{2}\right)\right\} \geq \min \left(\mathrm{T}\left(\mu_{\mathrm{A}}\left(\mathrm{x}_{1}\right), \mu_{\mathrm{A}}\left(\mathrm{y}_{1}\right)\right), \mathrm{T}\left(\mu_{\mathrm{A}}\left(\mathrm{x}_{2}\right), \mu_{\mathrm{A}}\left(\mathrm{y}_{2}\right)\right)\right) \geq \mathrm{T}\left(\min \left(\mu_{\mathrm{A}}\left(\mathrm{x}_{1}\right), \mu_{\mathrm{A}}\left(\mathrm{x}_{2}\right)\right), \min \left(\mu_{\mathrm{A}}\left(\mathrm{y}_{1}\right), \mu_{\mathrm{A}}\left(\mathrm{y}_{2}\right)\right.\right.$ )$\quad=\mathrm{T}\left(\mu_{\mathrm{V}}\left(\mathrm{x}_{1}, \mathrm{x}_{2}\right), \mu_{\mathrm{V}}\left(\mathrm{y}_{1}, \mathrm{y}_{2}\right)\right)=\mathrm{T}\left(\mu_{\mathrm{V}}(\mathrm{x}), \mu_{\mathrm{V}}(\mathrm{y})\right)$. Therefore, $\mu_{\mathrm{V}}(\mathrm{x}-\mathrm{y}) \geq \mathrm{T}\left(\mu_{\mathrm{V}}(\mathrm{x}), \mu_{\mathrm{V}}(\mathrm{y})\right)$, for all $\mathrm{x}, \mathrm{y}$ in F $\times F$. And, $\mu_{\mathrm{V}}\left(\mathrm{xy}^{-1}\right)=\mu_{\mathrm{V}}\left[\left(\mathrm{x}_{1}, \mathrm{x}_{2}\right)\left(\mathrm{y}_{1}, \mathrm{y}_{2}\right)^{-1}\right]=\mu_{\mathrm{V}}\left(\mathrm{x}_{1} \mathrm{y}_{1}{ }^{-1}, \mathrm{x}_{2} \mathrm{y}_{2}{ }^{-1}\right)=\min \left\{\mu_{\mathrm{A}}\left(\mathrm{x}_{1} \mathrm{y}_{1}{ }^{-1}\right), \mu_{\mathrm{A}}\left(\mathrm{x}_{2} \mathrm{y}_{2}{ }^{-1}\right)\right\} \geq \min ($ $\left.\mathrm{T}\left(\mu_{\mathrm{A}}\left(\mathrm{x}_{1}\right), \mu_{\mathrm{A}}\left(\mathrm{y}_{1}\right)\right), \mathrm{T}\left(\mu_{\mathrm{A}}\left(\mathrm{x}_{2}\right), \mu_{\mathrm{A}}\left(\mathrm{y}_{2}\right)\right)\right) \geq \mathrm{T}\left(\min \left(\mu_{\mathrm{A}}\left(\mathrm{x}_{1}\right), \mu_{\mathrm{A}}\left(\mathrm{x}_{2}\right)\right), \min \left(\mu_{\mathrm{A}}\left(\mathrm{y}_{1}\right), \mu_{\mathrm{A}}\left(\mathrm{y}_{2}\right)\right)\right)=\mathrm{T}\left(\mu_{\mathrm{V}}\left(\mathrm{x}_{1}, \mathrm{x}_{2}\right)\right.$, $\left.\mu_{\mathrm{V}}\left(\mathrm{y}_{1}, \mathrm{y}_{2}\right)\right)=\mathrm{T}\left(\mu_{\mathrm{V}}(\mathrm{x}), \mu_{\mathrm{V}}(\mathrm{y})\right)$. Therefore, $\mu_{\mathrm{V}}\left(\mathrm{xy}^{-1}\right) \geq \mathrm{T}\left(\mu_{\mathrm{V}}(\mathrm{x}), \mu_{\mathrm{V}}(\mathrm{y})\right)$, for all $\mathrm{x}$ and $\mathrm{y} \neq(0,0)$ in $\mathrm{F} \times \mathrm{F}$. Also we have, $v_{\mathrm{V}}(\mathrm{x}-\mathrm{y})=v_{\mathrm{V}}\left[\left(\mathrm{x}_{1}, \mathrm{x}_{2}\right)-\left(\mathrm{y}_{1}, \mathrm{y}_{2}\right)\right]=v_{\mathrm{V}}\left(\mathrm{x}_{1}-\mathrm{y}_{1}, \mathrm{x}_{2}-\mathrm{y}_{2}\right)=\max \left(v_{\mathrm{A}}\left(\mathrm{x}_{1}-\mathrm{y}_{1}\right), v_{\mathrm{A}}\left(\mathrm{x}_{2}-\mathrm{y}_{2}\right)\right) \leq \max ($ $\left.\mathrm{S}\left(v_{\mathrm{A}}\left(\mathrm{x}_{1}\right), v_{\mathrm{A}}\left(\mathrm{y}_{1}\right)\right), \mathrm{S}\left(v_{\mathrm{A}}\left(\mathrm{x}_{2}\right), v_{\mathrm{A}}\left(\mathrm{y}_{2}\right)\right)\right) \leq \mathrm{S}\left(\max \left(v_{\mathrm{A}}\left(\mathrm{x}_{1}\right), v_{\mathrm{A}}\left(\mathrm{x}_{2}\right)\right), \max \left(v_{\mathrm{A}}\left(\mathrm{y}_{1}\right), v_{\mathrm{A}}\left(\mathrm{y}_{2}\right)\right)\right)=\mathrm{S}\left(v_{\mathrm{V}}\left(\mathrm{x}_{1}\right.\right.$, $\left.\left.\mathrm{x}_{2}\right), v_{\mathrm{V}}\left(\mathrm{y}_{1}, \mathrm{y}_{2}\right)\right)=\mathrm{S}\left(v_{\mathrm{v}}(\mathrm{x}), v_{\mathrm{V}}(\mathrm{y})\right)$. Therefore, $v_{\mathrm{v}}(\mathrm{x}-\mathrm{y}) \leq \mathrm{S}\left(v_{\mathrm{v}}(\mathrm{x}), v_{\mathrm{V}}(\mathrm{y})\right)$, for all $\mathrm{x}$ and $\mathrm{y}$ in $\mathrm{F} \times \mathrm{F}$. And $v_{\mathrm{V}}\left(\mathrm{xy}^{-1}\right)=v_{\mathrm{V}}\left[\left(\mathrm{x}_{1}, \mathrm{x}_{2}\right)\left(\mathrm{y}_{1}, \mathrm{y}_{2}\right)^{-1}\right]=v_{\mathrm{V}}\left(\mathrm{x}_{1} \mathrm{y}_{1}^{-1}, \mathrm{x}_{2} \mathrm{y}_{2}^{-1}\right)=\max \left(v_{\mathrm{A}}\left(\mathrm{x}_{1} \mathrm{y}_{1}^{-1}\right), v_{\mathrm{A}}\left(\mathrm{x}_{2} \mathrm{y}_{2}^{-1}\right)\right) \leq \max \left(\mathrm{S}\left(\mathrm{v}_{\mathrm{A}}\left(\mathrm{x}_{1}\right), v_{\mathrm{A}}\left(\mathrm{y}_{1}\right)\right.\right.$ ), $\left.\mathrm{S}\left(v_{\mathrm{A}}\left(\mathrm{x}_{2}\right), v_{\mathrm{A}}\left(\mathrm{y}_{2}\right)\right)\right) \leq \mathrm{S}\left(\max \left(v_{\mathrm{A}}\left(\mathrm{x}_{1}\right), v_{\mathrm{A}}\left(\mathrm{x}_{2}\right)\right), \max \left(v_{\mathrm{A}}\left(\mathrm{y}_{1}\right), v_{\mathrm{A}}\left(\mathrm{y}_{2}\right)\right)\right)=\mathrm{S}\left(\mathrm{v}_{\mathrm{V}}\left(\mathrm{x}_{1}, \mathrm{x}_{2}\right), v_{\mathrm{V}}\left(\mathrm{y}_{1}, \mathrm{y}_{2}\right)\right)=$ $\mathrm{S}\left(v_{\mathrm{V}}(\mathrm{x}), v_{\mathrm{V}}(\mathrm{y})\right)$. Therefore, $v_{\mathrm{V}}\left(\mathrm{xy}^{-1}\right) \leq \mathrm{S}\left(v_{\mathrm{V}}(\mathrm{x}), v_{\mathrm{V}}(\mathrm{y})\right)$, for all $\mathrm{x}$ and $\mathrm{y} \neq(0,0)$ in $\mathrm{F} \times \mathrm{F}$. This proves that $\mathrm{V}$ is an intuitionistic (T, S)-fuzzy subfield of F $\times F$. Conversely, assume that $\mathrm{V}$ is an intuitionistic $(\mathrm{T}, \mathrm{S})$ fuzzy subfield of $F \times F$, then for any $x=\left(x_{1}, x_{2}\right)$ and $y=\left(y_{1}, y_{2}\right)$ are in $F \times F$, we have $\min \left\{\mu_{A}\left(x_{1}-y_{1}\right)\right.$, $\left.\mu_{\mathrm{A}}\left(\mathrm{x}_{2}-\mathrm{y}_{2}\right)\right\}=\mu_{\mathrm{V}}\left(\mathrm{x}_{1}-\mathrm{y}_{1}, \mathrm{x}_{2}-\mathrm{y}_{2}\right)=\mu_{\mathrm{V}}\left[\left(\mathrm{x}_{1}, \mathrm{x}_{2}\right)-\left(\mathrm{y}_{1}, \mathrm{y}_{2}\right)\right]=\mu_{\mathrm{V}}(\mathrm{x}-\mathrm{y}) \geq \mathrm{T}\left(\mu_{\mathrm{V}}(\mathrm{x}), \mu_{\mathrm{V}}(\mathrm{y})\right)=\mathrm{T}\left(\mu_{\mathrm{V}}\left(\mathrm{x}_{1}, \mathrm{x}_{2}\right), \mu_{\mathrm{V}}\left(\mathrm{y}_{1}\right.\right.$, $\left.\left.\mathrm{y}_{2}\right)\right)=\mathrm{T}\left(\min \left(\mu_{\mathrm{A}}\left(\mathrm{x}_{1}\right), \mu_{\mathrm{A}}\left(\mathrm{x}_{2}\right)\right), \min \left(\mu_{\mathrm{A}}\left(\mathrm{y}_{1}\right), \mu_{\mathrm{A}}\left(\mathrm{y}_{2}\right)\right)\right)$. If we put $\mathrm{x}_{2}=\mathrm{y}_{2}=0$, we get, $\mu_{\mathrm{A}}\left(\mathrm{x}_{1}-\mathrm{y}_{1}\right) \geq$ $\mathrm{T}\left(\mu_{\mathrm{A}}\left(\mathrm{x}_{1}\right), \mu_{\mathrm{A}}\left(\mathrm{y}_{1}\right)\right)$, for all $\mathrm{x}_{1}, \mathrm{y}_{1}$ in $\mathrm{F}$. And, $\min \left\{\mu_{\mathrm{A}}\left(\mathrm{x}_{1} \mathrm{y}_{1}{ }^{-1}\right), \mu_{\mathrm{A}}\left(\mathrm{x}_{2} \mathrm{y}_{2}{ }^{-1}\right)\right\}=\mu_{\mathrm{V}}\left(\mathrm{x}_{1} \mathrm{y}_{1}{ }^{-1}, \mathrm{x}_{2} \mathrm{y}_{2}{ }^{-1}\right)=\mu_{\mathrm{V}}\left[\left(\mathrm{x}_{1}, \mathrm{x}_{2}\right)\right.$ $\left.\left(\mathrm{y}_{1}, \mathrm{y}_{2}\right)^{-1}\right]=\mu_{\mathrm{V}}\left(\mathrm{xy}^{-1}\right) \geq \mathrm{T}\left(\mu_{\mathrm{V}}(\mathrm{x}), \mu_{\mathrm{V}}(\mathrm{y})\right)=\mathrm{T}\left(\mu_{\mathrm{V}}\left(\mathrm{x}_{1}, \mathrm{x}_{2}\right), \mu_{\mathrm{V}}\left(\mathrm{y}_{1}, \mathrm{y}_{2}\right)\right)=\mathrm{T}\left(\min \quad\left(\mu_{\mathrm{A}}\left(\mathrm{x}_{1}\right), \mu_{\mathrm{A}}\left(\mathrm{x}_{2}\right)\right), \min (\right.$ $\left.\mu_{\mathrm{A}}\left(\mathrm{y}_{1}\right), \mu_{\mathrm{A}}\left(\mathrm{y}_{2}\right)\right)$ ). If we put $\mathrm{x}_{2}=\mathrm{y}_{2}=1$, we get, $\mu_{\mathrm{A}}\left(\mathrm{x}_{1} \mathrm{y}_{1}^{-1}\right) \geq \mathrm{T}\left(\mu_{\mathrm{A}}\left(\mathrm{x}_{1}\right), \mu_{\mathrm{A}}\left(\mathrm{y}_{1}\right)\right)$, for all $\mathrm{x}_{1}$ and $\mathrm{y}_{1} \neq 0$ in $\mathrm{F}$. Also we have, $\max \left\{v_{\mathrm{A}}\left(\mathrm{x}_{1}-\mathrm{y}_{1}\right), v_{\mathrm{A}}\left(\mathrm{x}_{2}-\mathrm{y}_{2}\right)\right\}=v_{\mathrm{V}}\left(\mathrm{x}_{1}-\mathrm{y}_{1}, \mathrm{x}_{2}-\mathrm{y}_{2}\right)=v_{\mathrm{V}}\left[\left(\mathrm{x}_{1}, \mathrm{x}_{2}\right)-\left(\mathrm{y}_{1}, \mathrm{y}_{2}\right)\right]=v_{\mathrm{V}}(\mathrm{x}-\mathrm{y})$ $\leq \mathrm{S}\left(v_{\mathrm{V}}(\mathrm{x}), v_{\mathrm{V}}(\mathrm{y})\right)=\mathrm{S}\left(v_{\mathrm{V}}\left(\mathrm{x}_{1}, \mathrm{x}_{2}\right), v_{\mathrm{V}}\left(\mathrm{y}_{1}, \mathrm{y}_{2}\right)\right)=\mathrm{S}\left(\max \left(v_{\mathrm{A}}\left(\mathrm{x}_{1}\right), v_{\mathrm{A}}\left(\mathrm{x}_{2}\right)\right), \max \left(v_{\mathrm{A}}\left(\mathrm{y}_{1}\right), v_{\mathrm{A}}\left(\mathrm{y}_{2}\right)\right)\right)$. If we put $\mathrm{x}_{2}=$ $\mathrm{y}_{2}=0$, we get, $v_{\mathrm{A}}\left(\mathrm{x}_{1}-\mathrm{y}_{1}\right) \leq \mathrm{S}\left(\mathrm{v}_{\mathrm{A}}\left(\mathrm{x}_{1}\right), v_{\mathrm{A}}\left(\mathrm{y}_{1}\right)\right)$, for all $\mathrm{x}_{1}, \mathrm{y}_{1}$ in F. Also $\max \left\{v_{\mathrm{A}}\left(\mathrm{x}_{1} \mathrm{y}_{1}{ }^{-1}\right), v_{\mathrm{A}}\left(\mathrm{x}_{2} \mathrm{y}_{2}{ }^{-1}\right)\right\}=$ $v_{\mathrm{V}}\left(\mathrm{x}_{1} \mathrm{y}_{1}{ }^{-1}, \mathrm{x}_{2} \mathrm{y}_{2}^{-1}\right)=v_{\mathrm{V}}\left[\left(\mathrm{x}_{1}, \mathrm{x}_{2}\right)\left(\mathrm{y}_{1}, \mathrm{y}_{2}\right)^{-1}\right]=v_{\mathrm{V}}\left(\mathrm{xy}^{-1}\right) \leq \mathrm{S}\left(\mathrm{v}_{\mathrm{V}}(\mathrm{x}), v_{\mathrm{V}}(\mathrm{y})\right)=\mathrm{S}\left(\mathrm{v}_{\mathrm{V}}\left(\mathrm{x}_{1}, \mathrm{x}_{2}\right), v_{\mathrm{V}}\left(\mathrm{y}_{1}, \mathrm{y}_{2}\right)\right)=\mathrm{S}($ $\left.\max \left(v_{\mathrm{A}}\left(\mathrm{x}_{1}\right), v_{\mathrm{A}}\left(\mathrm{x}_{2}\right)\right), \max \left(v_{\mathrm{A}}\left(\mathrm{y}_{1}\right), v_{\mathrm{A}}\left(\mathrm{y}_{2}\right)\right)\right)$. If we put $\mathrm{x}_{2}=\mathrm{y}_{2}=1$, we get, $v_{\mathrm{A}}\left(\mathrm{x}_{1} \mathrm{y}_{1}^{-1}\right) \leq \mathrm{S}\left(\mathrm{v}_{\mathrm{A}}\left(\mathrm{x}_{1}\right), v_{\mathrm{A}}\left(\mathrm{y}_{1}\right)\right.$ ), for all $\mathrm{x}_{1}$ and $\mathrm{y}_{1} \neq 0$ in $\mathrm{F}$. Hence $\mathrm{A}$ is an intuitionistic (T, S)-fuzzy subfield of $\mathrm{F}$.

\section{Properties of Intuitionistic (T, S)-Fuzzy Subfields with Respect to the Homomorphism}

3.1 Theorem: Let $(\mathrm{F},+, \cdot)$ and $\left(\mathrm{F}^{\prime},+, \cdot\right)$ be any two fields. The homomorphic image of an intuitionistic (T, S)-fuzzy subfield of $\mathrm{F}$ is an intuitionistic (T, S)-fuzzy subfield of $\mathrm{F}^{\prime}$.

Proof: Let $(F,+, \cdot)$ and $\left(F^{\prime},+, \cdot\right)$ be any two fields and $f: F \rightarrow F^{\prime}$ be a homomorphism. That is $f(x+y)=$ $f(x)+f(y)$ for all $x$ and $y$ in $F, f(x y)=f(x) f(y)$, for all $x$ and $y$ in $F$. Let $V=f(A)$, where $A$ is an intuitionistic (T, S)-fuzzy subfield of $\mathrm{F}$. We have to prove that $\mathrm{V}$ is an intuitionistic (T, S)-fuzzy subfield of $\mathrm{F}^{\prime}$. Now, for $\mathrm{f}(\mathrm{x})$ and $\mathrm{f}(\mathrm{y})$ in $\mathrm{F}^{\prime}$, we have $\mu_{\mathrm{V}}(\mathrm{f}(\mathrm{x})-\mathrm{f}(\mathrm{y}))=\mu_{\mathrm{V}}(\mathrm{f}(\mathrm{x}-\mathrm{y})) \geq \mu_{\mathrm{A}}(\mathrm{x}-\mathrm{y}) \geq \mathrm{T}\left(\mu_{\mathrm{A}}(\mathrm{x})\right.$, $\left.\mu_{\mathrm{A}}(\mathrm{y})\right)$ which implies that $\mu_{\mathrm{V}}(\mathrm{f}(\mathrm{x})-\mathrm{f}(\mathrm{y})) \geq \mathrm{T}\left(\mu_{\mathrm{V}} \mathrm{f}(\mathrm{x})\right), \mu_{\mathrm{V}}(\mathrm{f}(\mathrm{y}))$, for all $\mathrm{f}(\mathrm{x})$ and $\mathrm{f}(\mathrm{y})$ in $\mathrm{F}^{\mathrm{l}}$. And $\mu_{\mathrm{V}}($ $\left.\mathrm{f}(\mathrm{x})(\mathrm{f}(\mathrm{y}))^{-1}\right)=\mu_{\mathrm{V}}\left(\mathrm{f}\left(\mathrm{xy}^{-1}\right)\right) \geq \mu_{\mathrm{A}}\left(\mathrm{xy}^{-1}\right) \geq \mathrm{T}\left(\mu_{\mathrm{A}}(\mathrm{x}), \mu_{\mathrm{A}}(\mathrm{y})\right)$ which implies that $\mu_{\mathrm{V}}\left(\mathrm{f}(\mathrm{x})(\mathrm{f}(\mathrm{y}))^{-1}\right) \geq \mathrm{T}($ $\left.\mu_{\mathrm{V}}(\mathrm{f}(\mathrm{x})), \mu_{\mathrm{V}}(\mathrm{f}(\mathrm{y}))\right)$, for all $\mathrm{f}(\mathrm{x})$ and $\mathrm{f}(\mathrm{y}) \neq 0^{1}$ in $\mathrm{F}^{\mathrm{l}}$. We have $v_{\mathrm{V}}(\mathrm{f}(\mathrm{x})-\mathrm{f}(\mathrm{y}))=v_{\mathrm{V}}(\mathrm{f}(\mathrm{x}-\mathrm{y})) \leq v_{\mathrm{A}}(\mathrm{x}-\mathrm{y}) \leq$ $\mathrm{S}\left(v_{\mathrm{A}}(\mathrm{x}), v_{\mathrm{A}}(\mathrm{y})\right)$ which implies that $v_{\mathrm{V}}(\mathrm{f}(\mathrm{x})-\mathrm{f}(\mathrm{y})) \leq \mathrm{S}\left(v_{\mathrm{V}}(\mathrm{f}(\mathrm{x})), v_{\mathrm{V}}(\mathrm{f}(\mathrm{y}))\right.$, for all $\mathrm{f}(\mathrm{x})$ and $\mathrm{f}(\mathrm{y})$ in $\mathrm{F}^{\prime}$. And $v_{\mathrm{V}}\left(\mathrm{f}(\mathrm{x})(\mathrm{f}(\mathrm{y}))^{-1}\right)=v_{\mathrm{V}}\left(\mathrm{f}\left(\mathrm{xy}^{-1}\right)\right) \leq v_{\mathrm{A}}\left(\mathrm{xy}^{-1}\right) \leq \mathrm{S}\left(v_{\mathrm{A}}(\mathrm{x}), v_{\mathrm{A}}(\mathrm{y})\right)$ which implies that $v_{\mathrm{V}}\left(\mathrm{f}(\mathrm{x})(\mathrm{f}(\mathrm{y}))^{-1}\right) \leq \mathrm{S}($ $\left.v_{\mathrm{V}}(\mathrm{f}(\mathrm{x})), v_{\mathrm{V}}(\mathrm{f}(\mathrm{y}))\right)$, for all $\mathrm{f}(\mathrm{x})$ and $\mathrm{f}(\mathrm{y}) \neq 0^{1}$ in $\mathrm{F}^{1}$. Hence $\mathrm{V}$ is an intuitionistic $(\mathrm{T}, \mathrm{S})$-fuzzy subfield of a field $\mathrm{F}^{\prime}$.

3.2 Theorem: Let $(\mathrm{F},+, \cdot)$ and $\left(\mathrm{F}^{1},+, \cdot\right)$ be any two fields. The homomorphic pre-image of an intuitionistic (T, S)-fuzzy subfield of $\mathrm{F}^{\prime}$ is an intuitionistic (T, S)-fuzzy subfield of $F$.

Proof: Let $(F,+, \cdot)$ and $\left(F^{\prime},+, \cdot\right)$ be any two fields and $f: F \rightarrow F^{\prime}$ be a homomorphism. That is $f(x+y)=$ $f(x)+f(y)$, for all $x$ and $y$ in $F$ and $f(x y)=f(x) f(y)$, for all $x$ and $y$ in $F$. Let $V=f(A)$, where $V$ is an intuitionistic (T, S)-fuzzy subfield of $F^{\prime}$. We have to prove that $A$ is an intuitionistic (T, S)-fuzzy subfield of $F$. Let $x$ and $y$ in $F$. Then $\mu_{\mathrm{A}}(\mathrm{x}-\mathrm{y})=\mu_{\mathrm{V}}(\mathrm{f}(\mathrm{x}-\mathrm{y}))=\mu_{\mathrm{V}}(\mathrm{f}(\mathrm{x})-\mathrm{f}(\mathrm{y})) \geq \mathrm{T}\left(\mu_{\mathrm{V}}(\mathrm{f}(\mathrm{x})), \mu_{\mathrm{V}}(\mathrm{f}(\mathrm{y}))\right)=$ $\mathrm{T}\left(\mu_{\mathrm{A}}(\mathrm{x}), \mu_{\mathrm{A}}(\mathrm{y})\right)$ which implies that $\mu_{\mathrm{A}}(\mathrm{x}-\mathrm{y}) \geq \mathrm{T}\left(\mu_{\mathrm{A}}(\mathrm{x}), \mu_{\mathrm{A}}(\mathrm{y})\right)$, for all $\mathrm{x}$ and $\mathrm{y}$ in $\mathrm{F}$. And, $\mu_{\mathrm{A}}\left(\mathrm{xy}^{-1}\right)=$ $\mu_{\mathrm{V}}\left(\mathrm{f}\left(\mathrm{xy}^{-1}\right)\right)=\mu_{\mathrm{V}}\left(\mathrm{f}(\mathrm{x})\left(\mathrm{f}\left(\mathrm{y}^{-1}\right)\right)=\mu_{\mathrm{V}}\left(\mathrm{f}(\mathrm{x})(\mathrm{f}(\mathrm{y}))^{-1}\right) \geq \mathrm{T}\left(\mu_{\mathrm{V}}(\mathrm{f}(\mathrm{x})), \mu_{\mathrm{V}}(\mathrm{f}(\mathrm{y}))\right)=\mathrm{T}\left(\mu_{\mathrm{A}}(\mathrm{x}), \mu_{\mathrm{A}}(\mathrm{y})\right)\right.$ which implies that $\mu_{\mathrm{A}}\left(\mathrm{xy}^{-1}\right) \geq \mathrm{T}\left(\mu_{\mathrm{A}}(\mathrm{x}), \mu_{\mathrm{A}}(\mathrm{y})\right)$, for all $\mathrm{x}$ and $\mathrm{y} \neq 0$ in $\mathrm{F}$. And, $v_{\mathrm{A}}(\mathrm{x}-\mathrm{y})=v_{\mathrm{V}}(\mathrm{f}(\mathrm{x}-\mathrm{y}))=v_{\mathrm{V}}($ $\mathrm{f}(\mathrm{x})-\mathrm{f}(\mathrm{y})) \leq \mathrm{S}\left(v_{\mathrm{V}}(\mathrm{f}(\mathrm{x})), v_{\mathrm{V}}(\mathrm{f}(\mathrm{y}))\right)=\mathrm{S}\left(v_{\mathrm{A}}(\mathrm{x}), v_{\mathrm{A}}(\mathrm{y})\right)$ which implies that $v_{\mathrm{A}}(\mathrm{x}-\mathrm{y}) \leq \mathrm{S}\left(v_{\mathrm{A}}(\mathrm{x}), v_{\mathrm{A}}(\mathrm{y})\right)$, for all $x$ and $y$ in F. And, $\left.v_{A}\left(x^{-1}\right)=v_{V}\left(f\left(x y^{-1}\right)\right)=v_{V}\left(f(x) f\left(y^{-1}\right)\right)=v_{V}\left(f(x)(f(y))^{-1}\right)\right) \leq S\left(v_{V}(f(x)), v_{V}(f(y))\right.$ 
)$=\mathrm{S}\left(v_{\mathrm{A}}(\mathrm{x}), v_{\mathrm{A}}(\mathrm{y})\right)$ which implies that $v_{\mathrm{A}}\left(\mathrm{xy} \mathrm{y}^{-1}\right) \leq \mathrm{S}\left(v_{\mathrm{A}}(\mathrm{x}), v_{\mathrm{A}}(\mathrm{y})\right)$, for all $\mathrm{x}, \mathrm{y} \neq 0$ in F. Hence $\mathrm{A}$ is an intuitionistic (T, S)-fuzzy subfield of a field $\mathrm{F}$.

3.3 Theorem: Let $A$ be an intuitionistic ( $T, S$ )-fuzzy subfield of a field $\mathrm{H}$ and $\mathrm{f}$ is an isomorphism from a field $\mathrm{F}$ onto $\mathrm{H}$. Then $\mathrm{A}$ of is an intuitionistic (T, S)-fuzzy subfield of $\mathrm{F}$.

Proof: Let $\mathrm{x}$ and $\mathrm{y}$ in $\mathrm{F}$ and $\mathrm{A}$ be an intuitionistic (T, S)-fuzzy subfield of a field $\mathrm{H}$. Then we have, $\left(\mu_{\mathrm{A}}^{\circ} \mathrm{f}\right)(\mathrm{x}-\mathrm{y})=\mu_{\mathrm{A}}(\mathrm{f}(\mathrm{x}-\mathrm{y}))=\mu_{\mathrm{A}}(\mathrm{f}(\mathrm{x})+\mathrm{f}(-\mathrm{y}))=\mu_{\mathrm{A}}(\mathrm{f}(\mathrm{x})-\mathrm{f}(\mathrm{y})) \geq \mathrm{T}\left(\mu_{\mathrm{A}}(\mathrm{f}(\mathrm{x})), \mu_{\mathrm{A}}(\mathrm{f}(\mathrm{y}))\right) \geq \mathrm{T}\left(\left(\mu_{\mathrm{A}} \circ \mathrm{f}\right)(\mathrm{x})\right.$, $\left.\left(\mu_{\mathrm{A}} \circ \mathrm{f}\right)(\mathrm{y})\right)$ which implies that $\left(\mu_{\mathrm{A}} \circ \mathrm{f}\right)(\mathrm{x}-\mathrm{y}) \geq \mathrm{T}\left(\left(\mu_{\mathrm{A}} \circ \mathrm{f}\right)(\mathrm{x}),\left(\mu_{\mathrm{A}} \circ \mathrm{f}\right)(\mathrm{y})\right)$, for all $\mathrm{x}$ and $\mathrm{y}$ in $\mathrm{F}$. And $\left(\mu_{\mathrm{A}} \circ \mathrm{f}\right)\left(\mathrm{xy}^{-1}\right)=\mu_{\mathrm{A}}\left(\mathrm{f}\left(\mathrm{xy} \mathrm{y}^{-1}\right)\right)=\mu_{\mathrm{A}}\left(\mathrm{f}(\mathrm{x}) \mathrm{f}\left(\mathrm{y}^{-1}\right)\right)=\mu_{\mathrm{A}}\left(\mathrm{f}(\mathrm{x})(\mathrm{f}(\mathrm{y}))^{-1}\right) \geq \mathrm{T}\left(\mu_{\mathrm{A}}(\mathrm{f}(\mathrm{x})), \mu_{\mathrm{A}}(\mathrm{f}(\mathrm{y}))\right) \geq \mathrm{T}\left(\left(\mu_{\mathrm{A}} \circ \mathrm{f}\right)(\mathrm{x}),\left(\mu_{\mathrm{A}} \circ \mathrm{f}\right.\right.$ )(y) ) which implies that $\left(\mu_{\mathrm{A}} \circ \mathrm{f}\right)\left(\mathrm{xy}^{-1}\right) \geq \mathrm{T}\left(\left(\mu_{\mathrm{A}} \circ \mathrm{f}\right)(\mathrm{x}),\left(\mu_{\mathrm{A}} \circ \mathrm{f}\right)(\mathrm{y})\right)$, for all $\mathrm{x}$ and $\mathrm{y} \neq 0$ in $\mathrm{F}$. We have, $\left(v_{\mathrm{A}}^{\circ} \circ\right)(\mathrm{x}-\mathrm{y})=v_{\mathrm{A}}(\mathrm{f}(\mathrm{x}-\mathrm{y}))=v_{\mathrm{A}}(\mathrm{f}(\mathrm{x})+\mathrm{f}(-\mathrm{y}))=v_{\mathrm{A}}(\mathrm{f}(\mathrm{x})-\mathrm{f}(\mathrm{y})) \leq \mathrm{S}\left(v_{\mathrm{A}}(\mathrm{f}(\mathrm{x})), v_{\mathrm{A}}(\mathrm{f}(\mathrm{y}))\right) \leq \mathrm{S}\left(\left(v_{\mathrm{A}} \circ \mathrm{f}\right)(\mathrm{x})\right.$, $\left.\left(v_{\mathrm{A}^{\circ}} \circ\right)(\mathrm{y})\right)$ which implies that $\left(v_{\mathrm{A}^{\circ}} \circ\right)(\mathrm{x}-\mathrm{y}) \leq \mathrm{S}\left(\left(v_{\mathrm{A}} \circ \mathrm{f}\right)(\mathrm{x}),\left(v_{\mathrm{A}}^{\circ} \circ\right)(\mathrm{y})\right)$, for all $\mathrm{x}$ and $\mathrm{y}$ in $\mathrm{F}$. And, $\left(v_{\mathrm{A}^{\circ}} \circ\right)(\mathrm{xy}$ $\left.{ }^{1}\right)=v_{A}\left(f\left(x y^{-1}\right)\right)=v_{A}\left(f(x) f\left(y^{-1}\right)\right)=v_{A}\left(f(x)(f(y))^{-1}\right) \leq S\left(v_{A}(f(x)), v_{A}(f(y))\right) \leq S\left(\left(v_{A} \circ f\right)(x),\left(v_{A} \circ f\right)(y)\right)$ which implies that $\left(v_{\mathrm{A}} \circ \mathrm{f}\right)\left(\mathrm{xy}^{-1}\right) \leq \mathrm{S}\left(\left(v_{\mathrm{A}} \circ \mathrm{f}\right)(\mathrm{x}),\left(v_{\mathrm{A}} \circ \mathrm{f}\right)(\mathrm{y})\right)$, for all $\mathrm{x}$ and $\mathrm{y} \neq 0$ in $\mathrm{F}$. Therefore $(\mathrm{A} \circ \mathrm{f})$ is an intuitionistic (T, S)-fuzzy subfield of a field $\mathrm{F}$.

3.4 Theorem: Let A be an intuitionistic (T, S)-fuzzy subfield of a field $(\mathrm{F},+,$.$) , then the pseudo$ intuitionistic $(\mathrm{T}, \mathrm{S})$-fuzzy coset $(\mathrm{aA})^{\mathrm{p}}$ is an intuitionistic $(\mathrm{T}, \mathrm{S})$-fuzzy subfield of a field $\mathrm{F}$, for every $\mathrm{a} \in \mathrm{F}$.

Proof: Let A be an intuitionistic (T, S)-fuzzy subfield of a field $(\mathrm{F},+$, . ). For every $\mathrm{x}$ and $\mathrm{y}$ in $\mathrm{F}$, we have, $\left(\left(a \mu_{\mathrm{A}}\right)^{\mathrm{p}}\right)(\mathrm{x}-\mathrm{y})=\mathrm{p}(\mathrm{a}) \mu_{\mathrm{A}}(\mathrm{x}-\mathrm{y}) \geq \mathrm{p}(\mathrm{a}) \mathrm{T}\left(\mu_{\mathrm{A}}(\mathrm{x}), \mu_{\mathrm{A}}(\mathrm{y})\right) \geq \mathrm{T}\left(\mathrm{p}(\mathrm{a}) \mu_{\mathrm{A}}(\mathrm{x}), \mathrm{p}(\mathrm{a}) \mu_{\mathrm{A}}(\mathrm{y})\right)=\mathrm{T}\left(\left(\left(\mathrm{a} \mu_{\mathrm{A}}\right)^{\mathrm{p}}\right)(\mathrm{x})\right.$, $\left.\left(\left(a \mu_{A}\right)^{p}\right)(y)\right)$. Therefore, $\quad\left(\left(a \mu_{A}\right)^{p}\right)(x-y) \geq T\left(\left(\left(a \mu_{A}\right)^{p}\right)(x),\left(\left(a \mu_{A}\right)^{p}\right)(y)\right)$, for all $x$ and $y$ in $F$. And for every $x$ and $y \neq 0$ in $F,\left(\left(a \mu_{A}\right)^{p}\right)\left(x y^{-1}\right)=p(a) \mu_{A}\left(x y^{-1}\right) \geq p(a) T\left(\mu_{A}(x), \mu_{A}(y)\right) \geq T\left(p(a) \mu_{A}(x), p(a) \mu_{A}(y)\right)=$ $\mathrm{T}\left(\left(\left(a \mu_{\mathrm{A}}\right)^{\mathrm{p}}\right)(\mathrm{x}),\left(\left(\mathrm{a} \mu_{\mathrm{A}}\right)^{\mathrm{p}}\right)(\mathrm{y})\right)$. Therefore, $\left(\left(\mathrm{a} \mu_{\mathrm{A}}\right)^{\mathrm{p}}\right)\left(\mathrm{xy} \mathrm{y}^{-1}\right) \geq \mathrm{T}\left(\left(\left(\mathrm{a} \mu_{\mathrm{A}}\right)^{\mathrm{p}}\right)(\mathrm{x}),\left(\left(\mathrm{a} \mu_{\mathrm{A}}\right)^{\mathrm{p}}\right)(\mathrm{y})\right)$, for all $\mathrm{x}$ and $\mathrm{y} \neq 0$ in F. We have, $\left(\left(a v_{A}\right)^{p}\right)(x-y)=p(a) v_{A}(x-y) \leq p(a) S\left(v_{A}(x), v_{A}(y)\right) \leq S\left(p(a) v_{A}(x), p(a) v_{A}(y)\right)=S\left(\left(\left(a v_{A}\right)^{p}\right.\right.$ )$\left.(x),\left(\left(a v_{A}\right)^{p}\right)(y)\right)$. Therefore, $\left(\left(a v_{A}\right)^{p}\right)(x-y) \leq S\left(\left(\left(a v_{A}\right)^{p}\right)(x),\left(\left(a v_{A}\right)^{p}\right)(y)\right)$, for all $x$ and $y$ in $F$. And for every $\mathrm{x}$ and $\mathrm{y} \neq 0$ in $\mathrm{F},\left(\left(\mathrm{a} v_{\mathrm{A}}\right)^{\mathrm{p}}\right)\left(\mathrm{xy}^{-1}\right)=\mathrm{p}(\mathrm{a}) v_{\mathrm{A}}\left(\mathrm{xy} \mathrm{y}^{-1}\right) \leq \mathrm{p}(\mathrm{a}) \mathrm{S}\left(v_{\mathrm{A}}(\mathrm{x}), v_{\mathrm{A}}(\mathrm{y})\right) \leq \mathrm{S}\left(\mathrm{p}(\mathrm{a}) v_{\mathrm{A}}(\mathrm{x}), \mathrm{p}(\mathrm{a}) v_{\mathrm{A}}(\mathrm{y})\right)=$ $\mathrm{S}\left(\left(\left(\mathrm{a} v_{\mathrm{A}}\right)^{\mathrm{p}}\right)(\mathrm{x}),\left(\left(\mathrm{a} v_{\mathrm{A}}\right)^{\mathrm{p}}\right)(\mathrm{y})\right)$. Therefore, $\left(\left(\mathrm{av}_{\mathrm{A}}\right)^{\mathrm{p}}\right)\left(\mathrm{xy}^{-1}\right) \leq \mathrm{S}\left(\left(\left(\mathrm{a} v_{\mathrm{A}}\right)^{\mathrm{p}}\right)(\mathrm{x}),\left(\left(\mathrm{a} v_{\mathrm{A}}\right)^{\mathrm{p}}\right)(\mathrm{y})\right)$, for all $\mathrm{x}$ and $\mathrm{y} \neq 0$ in $\mathrm{F}$. Hence $(\mathrm{aA})^{\mathrm{p}}$ is an intuitionistic $(\mathrm{T}, \mathrm{S})$-fuzzy subfield of a field $\mathrm{F}$.

\section{Reference}

[1] Akram. M and Dar.K.H, On fuzzy d-algebras, Punjab University Journal of Mathematics, 37, 61-76, (2005).

[2] Anthony.J.M. and Sherwood.H, Fuzzy groups Redefined, Journal of mathematical analysis and applications, $69,124-130$ (1979).

[3] Asok Kumer Ray, On product of fuzzy subgroups, Fuzzy sets and sysrems, 105, 181-183 (1999).

[4] Atanassov.K.T., Intuitionistic fuzzy sets, Fuzzy sets and systems, 20(1), 87-96 (1986).

[5] Atanassov.K.T., Intuitionistic fuzzy sets theory and applications, Physica-Verlag, A Springer-Verlag company, April 1999, Bulgaria.

[6] Azriel Rosenfeld, Fuzzy Groups, Journal of mathematical analysis and applications, 35, 512-517 (1971).

[7] Banerjee.B and D.K.Basnet,Intuitionistic fuzzy subrings and ideals, J.Fuzzy Math.11, no.1, 139-155 (2003).

[8] Biswas.R, Fuzzy subgroups and Anti-fuzzy subgroups, Fuzzy sets and systems, 35,121-124 ( 1990 ).

[9] Biswas.R, Fuzzy fields and fuzzy linear spaces redefined, Fuzzy sets and systems, (1989) North Holland.

[10] Davvaz.B, Wieslaw.A and Dudek, Fuzzy n-ary groups as a generalization of Rosen feld Fuzzy groups, ARXIV-0710. 3884VI (MATH.RA), 1-16, (20 OCT 2007 ).

[11] De, K., Biswas, R, Roy, A.R, On intuitionistic fuzzy sets, Notes on Intuitionistic Fuzzy Sets, 3(4), (1997).

[12] Hur.K, Kang.H.W and H.K.Song, Intuitionistic fuzzy subgroups and subrings, Honam Math. J. 25 no.1, 19-41 (2003).

[13] Hur.K, S.Y Jang and H.W Kang, 2005. (T, S)-intuitionistic fuzzy ideals of a ring, J.Korea Soc. Math.Educ.Ser.B: pure Appl.Math. 12(3): 193-209.

[14] Mustafa Akgul, Some properties of fuzzy groups, Journal of mathematical analysis and applications, 133, 93-100 (1988).

[15] Mohamed Asaad, Groups and fuzzy subgroups, fuzzy sets and systems (1991), North-Holland.

[16] Nanda. S, Fuzzy fields and fuzzy linear spaces, Fuzzy sets and systems, 19 (1986), 89-94.

[17] Palaniappan. N \& K.Arjunan, Some properties of intuitionistic fuzzy subgroups, Acta Ciencia Indica, Vol.XXXIII (2) : 321-328 (2007).

[18] Rajesh Kumar, Fuzzy Algebra, Volume 1, University of Delhi Publication Division, July -1993.

[19] Rosenfeld. A, Fuzzy groups, J. Math. Anal. Appl., 35 (1971), 512-517.

[20] Salah Abou-Zaid, On generalized characteristic fuzzy subgroups of a finite group, fuzzy sets and systems , 235-241 (1991).

[21] Vasantha kandasamy .W.B, Smarandache fuzzy algebra, American research press, Rehoboth -2003.

[22] Vasu.M, D.Sivakumar \& Arjunan. K, A study on anti (T, S)-fuzzy subfield of a field, Indian journal of Research, Vol. 1, Issue 1, 78-80 (2012).

[23] ZADEH.L.A, Fuzzy sets, Information and control, Vol.8, 338-353 (1965). 